\title{
Development of Molecular Markers Linked to the Allele Associated with the Non-astringent Trait of the Chinese Persimmon (Diospyros kaki Thunb.)
}

\author{
Ayako Ikegami $^{1 * *}$, Sai Eguchi ${ }^{1}$, Takashi Akagi ${ }^{1}$, Akihiko Sato $^{2}$, Masahiko Yamada $^{2 * * *}$, \\ Shinya Kanzaki ${ }^{3}$, Akira Kitajima ${ }^{4}$ and Keizo Yonemori ${ }^{1 *}$ \\ ${ }^{1}$ Graduate School of Agriculture, Kyoto University, Kyoto 606-8502, Japan \\ ${ }^{2}$ Grape and Persimmon Research Station, National Institute of Fruit Tree Science, National Agriculture and Food Research \\ Organization, Akitsu, Higashihiroshima 739-2494, Japan \\ ${ }^{3}$ Faculty of Agriculture, Kinki University, Nara 631-8505, Japan \\ ${ }^{4}$ Experimental Farm, Graduate School of Agriculture, Kyoto University, Takatsuki 569-0096, Japan
}

Persimmon cultivars are classified into four types depending on the nature of astringency loss of the fruit, namely pollination constant non-astringent (PCNA), pollination variant non-astringent (PVNA), pollination variant astringent (PVA), and pollination constant astringent (PCA). Among these four types, PCNA is the most important cultivar for persimmon breeding due to the stable loss of natural astringency on the tree. The trait of natural astringency loss is recessive in Japanese PCNA cultivars, while that in the Chinese PCNA cultivar, 'Luo tian tian shi', is dominant and the latter locus, termed $C P C N A$, is different from that in Japanese cultivars. In order to develop a molecular marker for the selection of the CPCNA-type cultivar, we performed amplified fragment length polymorphism (AFLP) in combination with bulked segregant analysis for $F_{1}$ offspring derived from ' $L u$ tian tian shi', which was used as the maternal parent. A total of 384 primer combinations were tested, and three AFLP markers, namely EACT-MCCC-222 (RO1), EGGC-MCTC-309 (RO2), and EGCC-MCGA-105 (RO3), linked to the CPCNA dominant allele were obtained. Among these markers, EGGC/MCTC-309 (RO2) was converted into a sequence-characterized amplified region (SCAR) marker. PCR analysis using $\mathrm{F}_{1}$ offspring $(\mathrm{n}=$ 264) revealed that the relevance ratio of the SCAR marker was $94 \%$. The polymorphism of the RO2 marker, which was strongly linked to the CPCNA dominant allele, was detected in only two Chinese PCNA cultivars, namely 'Luo tian tian shi' and 'Tian bao gai', among the Chinese, Korean, and Japanese cultivars tested. These results indicate that the $\mathrm{RO} 2$ marker contributes to marker-assisted selection for breeding programs of new PCNA cultivars having the $C P C N A$ trait.

Key Words: amplified fragment length polymorphism, astringency, bulked segregant analysis, Diospyros kaki, markerassisted selection.

\section{Introduction}

The cultivated persimmon $(2 n=6 x=90)$ fruit accumulates a large amount of condensed tannins/ proanthocyanidins (PAs), which cause astringency by coagulating proteins in taste buds and creating a

Received; May 13, 2010. Accepted; October 4, 2010.

* Corresponding author (E-mail: keizo@kais.kyoto-u.ac-jp).

** Present address: Department of Bioproduction Sciences, Ishikawa Prefectural University, Nonoichi 921-8836, Japan.

*** Present address: National Institute of Fruit Tree Science, National Agriculture and Food Research Organization, Tsukuba 305-8605, Japan. puckering or drying sensation. The fruits are classified as astringent (A) or non-astringent (NA) based on the loss of natural astringency on the tree. Astringent and non-astringent cultivars are further classified into pollination variant $(\mathrm{PV})$ or pollination constant based on the astringency loss and the presence of seeds (Hume, 1914). Thus, the fruits are classified into the following four types: pollination constant astringent (PCA), pollination constant non-astringent (PCNA), pollination variant astringent (PVA), and pollination variant nonastringent (PVNA) (Yonemori et al., 2000). In PV-type fruit, astringency loss is associated with volatiles, such as ethanol and acetaldehyde generated from seeds (Sugiura and Tomana, 1983). These volatiles promote 
insolubilization (polymerization) of PAs, which causes astringency loss in the persimmon fruit (Tanaka et al., 1994). Among these four types, PCNA fruit is the most desirable for fresh consumption because of the stable loss of astringency on the tree during fruit development (Yonemori et al., 2000). Astringent fruits accumulate PAs in the vacuoles of specific cells termed "tannin cells" and remain astringent until full maturation. In contrast, PCNA fruits terminate PA accumulation in these cells at an early stage of fruit development, thereby causing reduced PA levels and smaller tannin cells than those of non-PCNA (PCA, PVA, and PVNA) fruits (Yonemori and Matsushima, 1985, 1987).

PCNA-type persimmons were thought to have been developed uniquely in Japan; however, a PCNA cultivar, 'Luo tian tian shi' was discovered in China (Wang et al., 1982). The Japanese PCNA trait conferred by the AST locus is recessive in non-PCNA cultivars (Ikeda et al., 1985; Yamada and Sato, 2002). Since persimmon is hexaploid, crossing between Japanese PCNA and nonPCNA cultivars yielded only non-PCNA offspring, and backcrossing of this $\mathrm{F}_{1}$ individual with PCNA cultivars yielded only around $15 \%$ of PCNA offspring (Ikeda et al., 1985). In addition, the restricted gene pool for PCNA parents causes inbreeding depression. In contrast, crossing tests of Japanese PCNA or non-PCNA cultivars with Chinese PCNA cultivars yielded both astringent and non-astringent $F_{1}$ offspring in a nearly $1: 1$ ratio (Ikegami et al., 2004, 2006). This result suggests that the Chinese PCNA trait controlled by a locus, termed $C P C N A$, is dominant in non-PCNA cultivars; therefore, introducing CPCNA in PCNA cultivars in PCNA breeding programs is meaningful not only to expand the genetic background of PCNA breeding, but also to improve the occurrence ratio of PCNA in $\mathrm{F}_{1}$ offspring.

Marker-assisted selection (MAS) is considered a useful tool in breeding programs, especially for tree crops. Kanzaki et al. (2001) obtained restriction fragment length polymorphism (RFLP) markers linked to the $A S T$ locus by performing amplified fragment length polymorphism (AFLP) in combination with bulked segregant analysis (BSA), and these markers were later converted into PCR markers (Kanzaki et al., 2009). To develop an MAS system of CPCNA for breeding Chinese-type PCNA persimmons, we performed AFLP analysis (Vos et al., 1995) in combination with BSA (Michelmore et al., 1991).

\section{Materials and Methods}

\section{Plant materials}

Leaves of the $F_{1}$ population derived from a cross between Chinese PCNA 'Luo tian tian shi' and Japanese PCNA 'Okugosho' (RO family) were used for AFLP analysis. The segregation ratio of PCNA/non-PCNA in RO family is $48: 56(n=102)$. As 'Luo tian tian shi' does not have the ast allele (Akagi et al., 2010; Kanzaki et al., 2000a), $F_{1}$ plants derived from crosses between
'Luo tian tian shi' and Japanese PCNA cultivars are heterozygous on the locus (Ikegami et al., 2006); therefore, the PCNA phenotype of offspring segregated in the $\mathrm{F}_{1}$ population of the cross is not controlled by the ast allele, but the CPCNA allele. Leaf samples were obtained from seedlings top-grafted and grown in the field of the Grape and Persimmon Research Station, National Institute of Fruit Tree Science, Higashihiroshima, Japan, or from seedlings planted directly into the ground at the Experimental Farm, Kyoto University, Takatsuki, Japan. BSA was performed by pooling DNA of 10 PCNA plants as a PCNA bulk and 10 non-PCNA plants as a nonPCNA bulk.

For evaluation of the SCAR marker, $264 \mathrm{~F}_{1}$ offspring of 'Luo tian tian shi' and other different cultivars were analyzed. $F_{1}$ offspring of 'Luo tian tian shi' were as follows: $81 \mathrm{~F}_{1}$ offspring of RO family; $98 \mathrm{~F}_{1}$ offspring of 'Luo tian tian shi' $\times$ PCA 'Yotsumizo' (RY family); $49 \mathrm{~F}_{1}$ offspring of 'Luo tian tian shi' $\times$ PCA 'Iwasedo' (RI family); and $36 \mathrm{~F}_{1}$ offspring of 'Luo tian tian shi' $\times$ Japanese PCNA 'Taishu' (310 family), and the cultivars were as follows: two Chinese PCNA cultivars, 'Luo tian tian shi' and 'Tian bao gai'; five Japanese PCNA cultivars, 'Fuyu', 'Gosho', 'Jiro', 'Hanagosho', and 'Taishu'; five Japanese non-PCNA cultivars, 'Amahyakume', 'Atago', 'Iwasedo', 'Saijo', and 'Yotsumizo'; and nine Chinese and Korean non-PCNA cultivars, 'Ban si' (Korea), 'Sa kok si' (Korea), 'Jin shi' (China), 'Huo jing' (China), 'Huo shi' (China), 'Qu jing shui shi' (China), 'Da mo pan' (China), 'Hei xin shi' (China), and 'Mei xian niu xin shi' (China). 'Tian bao gai' is reported to be a PCNA cultivar found in Luo tian County, Hubei province, China, according to sensory testing and analysis of tannin cell size (Yonemori et al., 2005). All cultivars used were conserved and grown in Akitsu, Grape and Persimmon Research Station, National Institute of Fruit Tree Science.

\section{AFLP analysis}

Total genomic DNA was extracted from the leaves of each plant by the CTAB method according to Doyle and Doyle (1987). After purifying DNA by phenol extraction and polyethylene glycol precipitation, DNA concentration was adjusted to $50 \mathrm{ng} \cdot \mu \mathrm{L}^{-1}$ for AFLP analysis. DNA digestion, adapter ligation, and preamplification were performed as described by Vos et al. (1995) and Kanzaki et al. (2001) using the AFLP analysis system I kit (Invitrogen, USA). Genomic DNA $(1.25 \mu \mathrm{g})$ of each bulked sample, parent, and individual was digested with EcoRI and $M s e I$ in $12.5 \mu \mathrm{L}$ reaction mixture, and EcoRI and $M s e I$ adapters for each restriction site were ligated using T4 DNA ligase, diluted 10 times with TE buffer, and then used as template DNA. After preamplification with $2.5 \mu \mathrm{L}$ template DNA in a total of $25 \mu \mathrm{L}$ reaction volume, selective amplification was performed with primer pairs including three additional selective nucleotides at the 3' end of each primer. A total of 384 
combinations of EcoRI and MseI primers were used for screening PCNA and non-PCNA bulks (Table 1). PCR products of selective amplifications were denatured by boiling with an equal volume of the loading dye ( $98 \%$ formamide, $10 \mathrm{mM}$ ethylenediaminetetraacetic acid, $0.05 \%$ bromophenol blue, and $0.05 \%$ xylene cyanol), and aliquots were loaded onto $6 \%$ polyacrylamide gel. After electrophoresis, fragments were transferred onto the Biodine B membrane (Pall, USA). The membrane was then hybridized with $5^{\prime}$ digoxigenin (DIG)-labeled oligonucleotide (Sigma Genosis, Japan), which hybridizes with the EcoRI adaptor sequence at $42^{\circ} \mathrm{C}$ for $2 \mathrm{~h}$. After hybridization, membranes were first washed twice in $2 \times \mathrm{SSC}$ containing $0.1 \% \mathrm{SDS}$ for $5 \mathrm{~min}$ at room temperature, and then twice in $0.1 \times \mathrm{SSC}$ containing $0.1 \% \mathrm{SDS}$ for $15 \mathrm{~min}$ at $42^{\circ} \mathrm{C}$. Target bands were detected using anti-DIG-alkaline phosphatase (Roche Applied Science, USA) and chemiluminescent substrate CDPstar (Roche Applied Science) according to the manufacturer's instructions.

\section{Cloning of AFLP markers}

Three AFLP markers (RO1, RO2, and RO3) were detected as candidates, and were recovered from gels electrophoresed and stained using the Silver Sequence ${ }^{\mathrm{TM}}$ DNA sequencing system (Promega, USA). Staining was performed according to the manufacturer's instructions. TE buffer $(150 \mu \mathrm{L})$ was added to each excised marker band, and the bands were frozen and thawed at $-20^{\circ} \mathrm{C}$ and $4^{\circ} \mathrm{C}$, respectively. After denaturing by incubation for $15 \mathrm{~min}$ at $95^{\circ} \mathrm{C}$, samples were centrifuged at $15,000 \mathrm{rpm}$ for $5 \mathrm{~min}$ at $4^{\circ} \mathrm{C}$. The supernatant was then transferred to a fresh tube and precipitated with ethanol, dried in a vacuum, and dissolved in $10 \mu \mathrm{L}$ TE buffer. After $10 \mathrm{ng}$ of the recovered DNA was reamplified with the same selective primer combination, the reamplified product was subcloned into the pGEM-T easy vector (Promega). DNA sequences of the resultant plasmids were determined with $\mathrm{T} 7$ or M13RV primers using the DYEnamic ET terminator cycle sequencing kit (GE Healthcare, USA) and ABI PRISM 310 DNA genetic analyzer (Applied Biosystems, USA).

Table 1. EcoRI and MseI primers used for bulked segregant analysis.

\begin{tabular}{llllll}
\hline \hline & \multicolumn{3}{c}{ EcoRI primers } & & \multicolumn{2}{c}{ MseI primers } \\
\cline { 1 - 2 } \cline { 5 - 6 } EAAG & EGAA & EGGA & & MCAA & MCGA \\
EAAC & EGAT & EGGT & & MCAT & MCGT \\
EAGG & EGAG & EGGG & & MCAG & MCGG \\
EAGC & EGAC & EGGC & & MCAC & MCGC \\
EACA & EGTA & EGCA & & MCTA & MCCA \\
EACT & EGTT & EGCT & & MCTT & MCCT \\
EACG & EGTG & EGCG & & MCTG & MCCG \\
EACC & EGTC & EGCC & & MCTC & MCCC \\
\hline
\end{tabular}

\section{RFLP analysis}

Total genomic DNA $(20 \mu \mathrm{g})$ from 'Luo tian tian shi', 'Okugosho', and three offspring of PCNA or non-PCNA was digested with restriction enzymes Hind III or XbaI, run onto $0.8 \%$ agarose gel, transferred onto a Biodyne Plus membrane (Pall), and hybridized with a DIGlabeled probe prepared using the PCR DIG probe synthesis kit (Roche Applied Science). The probe was prepared using the AFLP fragment cloned into the pGEM-T easy vector as a template and primer pairs (forward: 5'-GAGTCCTGAGTAACCCCGCATT-3', reverse: 5'-CGAGTAGTGTTGGAGTCAGAGT-3' for RO1; forward: 5'-TCTTTACAATCGCGCCGGGGGG3', reverse: 5'-ACTCATGTTCGTAACAATGGCC-3' for RO2; forward: 5'-GTACCAATTCGCCTGACTGAAA3', reverse: 5'-TCCTGAGTAACGACGTCACACT-3' for RO3). After hybridization of membranes at $64^{\circ} \mathrm{C}(\mathrm{RO} 1)$, $63.5^{\circ} \mathrm{C}(\mathrm{RO} 2)$, and $55^{\circ} \mathrm{C}(\mathrm{RO} 3)$ for $16 \mathrm{~h}$, the blot was first washed twice in $2 \times \mathrm{SSC}$ containing $0.1 \% \mathrm{SDS}$ for $5 \mathrm{~min}$ at $64^{\circ} \mathrm{C}(\mathrm{RO} 1), 63.5^{\circ} \mathrm{C}(\mathrm{RO} 2)$, and $55^{\circ} \mathrm{C}(\mathrm{RO} 3)$, and then twice in $0.1 \times \mathrm{SSC}$ containing $0.1 \% \mathrm{SDS}$ for $15 \mathrm{~min}$ at $64^{\circ} \mathrm{C}(\mathrm{RO} 1), 63.5^{\circ} \mathrm{C}(\mathrm{RO} 2)$, and $55^{\circ} \mathrm{C}(\mathrm{RO} 3)$. Target signals were detected as described for AFLP analysis.

\section{Evaluation of the SCAR marker}

A SCAR marker was designed from the $\mathrm{RO} 2$ sequence. The PCR reaction mixture consisted of $10 \mathrm{ng}$ template DNA, $1 \times$ reaction buffer (supplied with Takara rTaq polymerase; Takara Bio, Japan), $200 \mu \mathrm{M}$ dNTPs, $0.2 \mu \mathrm{M}$ of a designed primer pair (2-2F: 5'-TCTT TACAACTGAGAAGGGGGG-3', 2-1R: 5'-TGTCTTA CAGACGTCGTCGGT-3'), and $1 \mathrm{U}$ rTaq DNA polymerase (Takara Bio) in $15 \mu \mathrm{L}$ reaction volume. A designed primer (2-1F: 5'-CGAAGTAATGTGCCGGTA AGCT-3') in place of 2-2F was used as a positive control reaction for non-PCNA-type (see Fig.3). The PCR reaction with a primer pair (2-1F and 2-2R) gives a 122bp band both in PCNA and non-PCNA types. The mixture was initially denatured at $95^{\circ} \mathrm{C}$ for $1 \mathrm{~min}$, followed by 35 cycles of PCR amplification with denaturation for $20 \mathrm{~s}$ at $94^{\circ} \mathrm{C}$, primer annealing for $20 \mathrm{~s}$ at $58.5^{\circ} \mathrm{C}$, and primer extension for $30 \mathrm{~s}$ at $72^{\circ} \mathrm{C}$. Amplified products were separated by electrophoresis on $2 \%$ agarose gel with $1 \times$ TAE buffer and stained with ethidium bromide. This SCAR marker was tested for $264 \mathrm{~F}_{1}$ offspring and cultivars (see Plant materials). Based on the presence or absence of the 158-bp band linked to the Chinese PCNA (CPCNA) trait, the marker genotype of each $F_{1}$ offspring was determined. The phenotype of the fruit was determined based on two parameters deeply related to proanthocyanidin accumulation, tannin cell size, and soluble phenol content, as described in previous reports (Ikegami et al., 2006). The non-PCNA type has larger tannin cells and higher soluble phenol content compared to the PCNA type. The normal distribution of PCNA/non-PCNA in tannin cell size and 
soluble phenol content slightly overlaps; therefore, the assessment of the phenotype may not be precise. However, the trait appears to be independent and no individual showed intermediate tannin cell size and soluble phenol content among the $F_{1}$ offspring tested in this analysis.

\section{Results}

A total of 384 primer combinations of $24 E c o R I$ and $16 \mathrm{Mse}$ I primers were used in BSA against subsets of PCNA and non-PCNA bulks. Ninety-four polymorphic markers present in PCNA and absent in non-PCNA were identified between the two bulks. After these markers were tested using the same individual plants used for bulks and parents, three primer sets (EACT-MCCC, EGGC-MCTC, and EGCC-MCGA) used for AFLP markers and designated RO1 (222 bp), RO2 (309 bp), and RO3 (105 bp), respectively, were present in PCNA but not in non-PCNA offspring (Fig. 1). Sequences of these three fragments (RO1, RO2, and RO3) were determined and used as probes in the following RFLP analysis. RFLP analyses in 'Okugosho', 'Luo tian tian shi', and six $\mathrm{F}_{1}$ offspring showed multiple bands in RO1 and RO3; however, only a single band specific to PCNA was detected in $\mathrm{RO} 2$ (Fig. 2).

In all RFLP analyses, PCNA-specific polymorphisms were detected (Fig. 2, arrows indicate a PCNA-specific signal). This result suggests that the three AFLP markers are applicable as RFLP markers for distinguishing the PCNA type for the progeny. In order to apply these three markers to a large population, we tried to develop PCRbased markers. Among these markers, the RO2 marker was easily converted to the SCAR marker. As for RO1
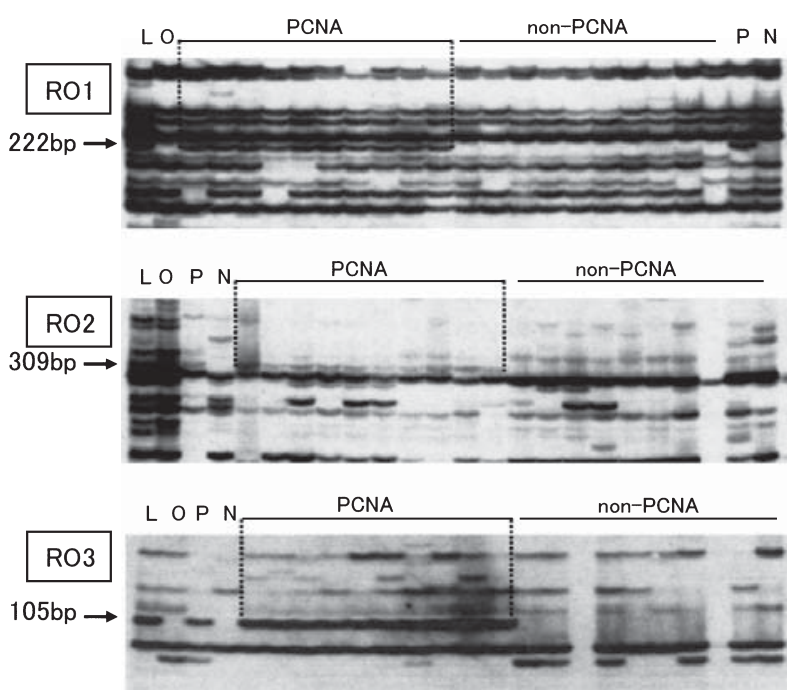

Fig. 1. AFLP fingerprints of PCNA and non-PCNA bulks, parents, and individuals for performing bulked segregant analysis (BSA) using primer combination EACT/MCCC-222 (RO1), EGGCMCTC-309 (RO2), and EGCC-MCGA-105 (RO3). Lanes: L, 'Luo tian tian shi'; O, 'Okugosho'; P, PCNA bulk; N, non-PCNA bulk; PCNA, PCNA individuals; non-PCNA, non-PCNA individuals. Arrows indicate AFLP markers and their sizes. and RO3, SCAR markers were also developed using the flanking sequences in the fosmid clones screened in a 'Luo tian tian shi' genomic library; however, the relevance rate of these markers was lower than that of RO2 (T. Akagi, unpublished data). Sequences of the AFLP fragment and a primer set of the SCAR marker for $\mathrm{RO} 2$ are given in Figure 3. The resultant SCAR marker for $\mathrm{RO} 2$ was a dominant marker linked to the CPCNA allele, and amplified the PCNA-specific 158bp fragment (Fig. 4A), while the 122-bp band with 2-1F and 2-1R was detected both in PCNA and non-PCNA types as a positive control (data not shown). Interestingly, this SCAR marker fragment was detected not only in 'Luo tian tian shi' but also in 'Tian bao gai' (Fig. 4B). Furthermore, the fact that the 158-bp fragment of the SCAR marker for RO2 was not detected in all Japanese PCNA cultivars tested (Fig. 4B) suggests that the nonastringent trait of all Japanese PCNA cultivars is due to homozygous recessive alleles on the $A S T$ locus, which is different from alleles on the CPCNA locus.

PCR analysis using $\mathrm{F}_{1}$ offspring $(\mathrm{n}=264)$ revealed that the average relevance rate of the SCAR marker was 94\% (Table 2). In the three $\mathrm{F}_{1}$ families, 'Luo tian tian shi' $\times$ 'Okugosho', 'Luo tian tian shi' $\times$ 'Yotsumizo', and

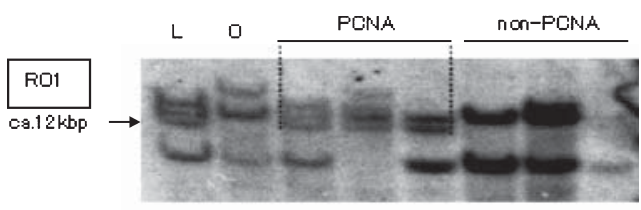

$X b a r$

digestion

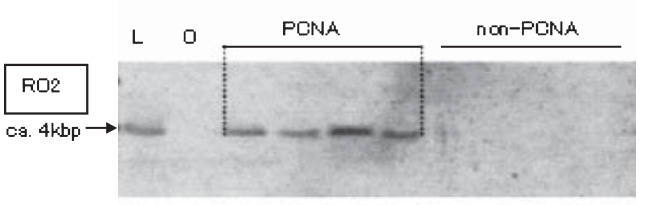

Xba

digestion

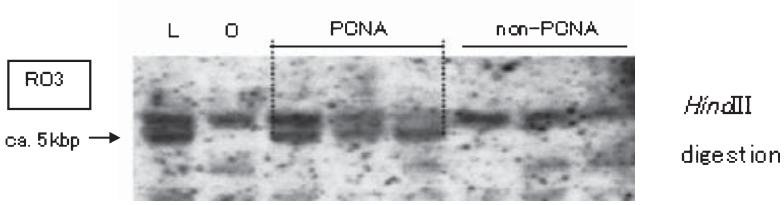

Fig. 2. RFLP analysis of genomic DNA digested with $X b a \mathrm{I}$ for RO1 and RO2, and HindIII for RO3. Lanes: L, 'Luo tian tian shi'; $\mathrm{O}$, 'Okugosho'; PCNA, PCNA offspring; non-PCNA, nonPCNA offspring. Arrows indicate RFLP markers and their sizes.

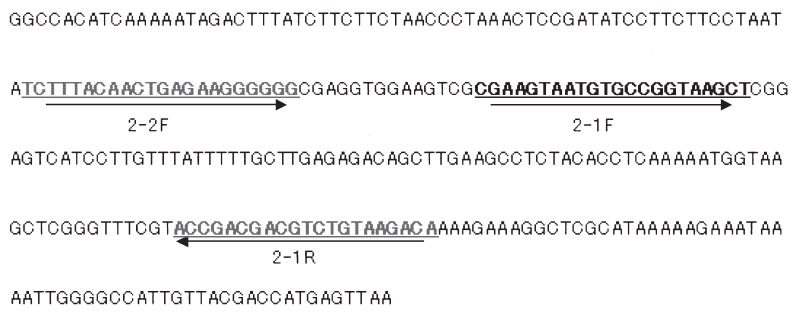

Fig. 3. Sequence of the AFLP fragment for $\mathrm{RO} 2$ and the primer set designed for SCAR. 
'Luo tian tian shi' $\times$ 'Iwasedo', the relevance rate were $96.3 \%, 94.9 \%$, and $95.9 \%$; thus, the occurrence rates of the recombination type were $3.7 \%, 5.1 \%$, and $4.1 \%$, respectively. The relevance rate in 'Luo tian tian shi' $\times$ 'Taishu' was $83.3 \%$, which was lower than that of the other $F_{1}$ families. This result was presumably due to the small population of the latter family $(n=36)$. Our results clearly show that the SCAR marker for RO2 is strongly linked to the CPCNA allele and that it will greatly contribute to MAS.

\section{Discussion}

To our knowledge, this is the first study on genomic analysis of the CPCNA allele. Previous reports on the inheritance of the PCNA phenotype in 'Luo tian tian shi' (Ikegami et al., 2004, 2006) suggest that the Chinese PCNA phenotype is controlled by a locus different from the PCNA phenotype of Japanese cultivars. In addition, 'Luo tian tian shi' is suggested to be a phylogenetically distant relative of Japanese PCNA cultivars, as demonstrated by AFLP analysis (Kanzaki et al., 2000b). The SCAR marker designed from the RO2 sequence
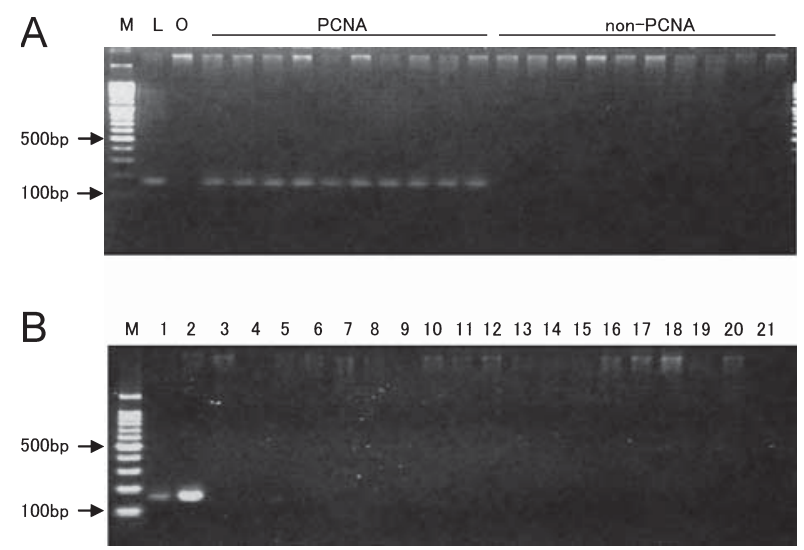

Fig. 4. $P C R$ analysis of markers in the $F_{1}$ population (A) and cultivars including Chinese PCNA cultivar 'Tian bao gai' (B). A. Lanes: M, 100-bp ladder marker; L, 'Luo tian tian shi'; O, 'Okugosho'; PCNA, PCNA individuals; non-PCNA, non-PCNA individuals. B. Lanes: M, 100-bp ladder marker; 1, 'Luo tian tian shi'; 2, 'Tian bao gai'; 3, 'Fuyu'; 4, 'Gosho'; 5, 'Jiro'; 6, 'Hanagosho'; 7, 'Taishu'; 8, 'Amahyakume'; 9, 'Atago'; 10, 'Iwasedo'; 11, 'Saijo'; 12, 'Yotsumizo'; 13, 'Ban si'; 14, 'Sa kok si'; 15, 'Jin shi'; 16,'Huo jing'; 17, 'Huo shi'; 18, 'Qu jing shui shi'; 19, 'Da mo pan'; 20, 'Hei xin shi'; 21, 'Mei xian niu xin shi' (see plant materials). was detected only in 'Luo tian tian shi' and 'Tian bao gai' among the cultivars tested in this study. The genetic background of 'Tian bao gai' with respect to the nonastringent trait has not been explained to date. Our results imply that this trait in 'Tian bao gai' is due to the same dominant allele on the CPCNA locus as in 'Luo tian tian shi'. The study also showed a high specificity of RO2 polymorphism linked to the dominant allele on the CPCNA locus among cultivars. Analysis using $\mathrm{F}_{1}$ offspring $(\mathrm{n}=264)$ also revealed that the recombination value between the SCAR marker and CPCNA allele was around $6 \%$; therefore, the SCAR marker for $\mathrm{RO} 2$ would enable MAS for the PCNA phenotype derived from the CPCNA dominant allele.

Determining the molecular function of the $C P C N A$ locus or CPCNA dominant allele would be interesting. In Chinese PCNA-type fruits, most of the expression of genes involved in PA biosynthesis, such as anthocyanidin reductase and dihydroflavonol 4-reductase, does not markedly decrease as in Japanese PCNA-type fruits (Ikegami et al., 2005), but genes whose functions in PA biosynthesis remain unknown, such as the genes encoding serine carboxypeptidase-like proteins, 3-dehydroquinate dehydratase/shikimate 5-dehydrogenase, glutathione $S$-transferase, and flavonoid 3-galactosyltransferase, decrease coincidentally with the PA level (Ikegami et al., 2009). Identification of this CPCNA locus should reveal new aspects of the regulation of biosynthesis of plant flavonoids or secondary metabolites. The dominant character of the CPCNA trait suggests that an inhibitorlike function in PA accumulation would exist on this locus (or dominant allele). In this study, we identified three AFLP markers linked to the CPCNA allele, which would help identify the CPCNA allele by map-based positional cloning. Ikegami et al. (2006) reported that the segregation ratio of PCNA and non-PCNA was nearly $1: 1$ in the $\mathrm{F}_{1}$ offspring used in this study, and that 'Luo tian tian shi' seemed to be simplex at the CPCNA locus, suggesting the following three allelic statuses for the CPCNA locus: Aaaaaa (autohexaploid), Aaaa (autoallohexaploid), and Aa (autoallohexaploid or allohexaploid). Identifying a locus showing the polyploidy inheritance mode on a genetic map is difficult; however, genomewide mapping has recently been performed using AFLP markers in the hexaploid sweet potato (Ipomoea batatas)

Table 2. Relevance ratios of the SCAR marker developed from the AFLP marker RO2 in $F_{1}$ offspring of 'Luo tian tian shi'.

\begin{tabular}{lcccc}
\hline \hline Population name (pollen parent) & RO (Okugosho) & RY (Yotsumizo) & RI (Iwasedo) & 310 (Taishu) \\
\hline Number of individuals tested $^{\mathrm{z}}$ & 81 & 98 & 49 & 36 \\
Number of individuals matched $^{\mathrm{y}}$ & 78 & 93 & 47 & 30 \\
Relevance ratio $^{\mathrm{x}}$ & 0.96 & 0.95 & 0.96 & 0.83 \\
\hline
\end{tabular}

z PCNA/non-PCNA phenotype of each individual was confirmed based on tannin cell size and soluble phenol content.

${ }^{y}$ Marker genotype determined by PCR was evaluated for each individual PCNA or non-PCNA phenotype.

${ }^{\mathrm{x}}$ Ratio of individuals matched in individuals tested. 
(Cervantes-Flore et al., 2008a, b; Kriegner et al., 2003). Furthermore, Akagi et al. (2009, 2010) determined the allelic status in the $A S T$ locus, which contains six $A S T /$ ast alleles, using quantitative real-time PCR analysis in the hexaploid persimmon. In the future, the three AFLP markers isolated in this study and the above-mentioned new techniques for genetic analysis of polyploid plants would contribute to identification of the $C P C N A$ locus in Chinese PCNA persimmon.

\section{Literature cited}

Akagi, T., S. Kanzaki, M. Gao, R. Tao, D. E. Parfitt and K. Yonemori. 2009. Quantitative real-time PCR to determine allele number for the astringency locus by analysis of a linked marker in Diospyros kaki Thunb. Tree Genet. Genomes 5: 483-492.

Akagi, T., Y. Takeda, A. Ikegami, A. Kono, A. Yamada, S. Kanzaki and K. Yonemori. 2010. Quantitative genotyping for astringency locus in hexaploid persimmon cultivars using quantitative real-time PCR. J. Amer. Soc. Hort. Sci. 135: 5966.

Cervantes-Flores, J. C., G. C. Yencho, A. Kriegner, K. V. Pecota, M. A. Faulk, R. O. M. Mwanga and B. Sosinski. 2008a. Development of a genetic linkage map and identification of homologous linkage groups in sweetpotato using multipledose AFLP markers. Mol. Breeding 21: 511-532.

Cervantes-Flores, J. C., G. C. Yencho, K. V. Pecota, B. Sosinski and R. O. M. Mwanga. 2008b. Detection of quantitative trait loci and inheritance of root-knot nematode resistance in sweetpotato. J. Amer. Soc. Hort. Sci. 133: 844-851.

Doyle, J. J. and J. L. Doyle. 1987. A rapid DNA isolation procedure for small quantities of flesh leaf tissue. Phytochem. Bul. 19: 11 .

Hume, H. H. 1914. A kaki classification. J. Hered. 5: 400-406.

Ikeda, I., M. Yamada, A. Kurihara and T. Nishida. 1985. Inheritance of astringency in Japanese persimmon. J. Japan. Soc. Hort. Sci. 54: 39-45 (In Japanese with English abstract).

Ikegami, A., T. Akagi, D. Potter, M. Yamada, A. Sato, K. Yonemori, A. Kitajima and K. Inoue. 2009. Molecular identification of 1-Cys peroxiredoxin and anthocyanin/flavonol 3-Ogalactosyltransferase from proanothocyanidin-rich young fruit of persimmon (Diospyros kaki Thunb.). Planta 230: 841855.

Ikegami, A., S. Eguchi, K. Yonemori, M. Yamada, A. Sato, N. Mitani and A. Kitajima. 2006. Segregations of astringent progenies in the $\mathrm{F}_{1}$ populations derived from crosses between a Chinese pollination-constant, non-astringent (PCNA) 'Luo Tain Tian Shi', and Japanese PCNA and pollination-constant, astringent (PCA) cultivars of Japanese origin. HortScience 41: 561-563.

Ikegami, A., K. Yonemori, A. Kitajima, A. Sato and M. Yamada. 2005. Expression of genes involved in proanthocyanidin biosynthesis during fruit development in a Chinese pollination-constant, nonastringent (PCNA) persimmon, 'Luo Tian Tian Shi'. J Amer. Soc. Hort. Sci. 130: 830-835.

Ikegami, A., K. Yonemori, A. Sugiura, A. Sato and M. Yamada. 2004. Segregation of astringency in $F_{1}$ progenies derived from crosses between pollination-constant, nonastringent persimmon cultivars. HortScience 39: 371-374.
Kanzaki, S., M. Yamada, A. Sato, N. Mitani, N. Utsunomiya and K. Yonemori. 2009. Conversion of RFLP markers for the selection of pollination-constant and non-astringent type persimmons (Diospyros kaki Thunb.) into PCR-based markers. J. Japan. Soc. Hort. Sci. 78: 68-73.

Kanzaki, S., K. Yonemori, A. Sato, M. Yamada and A. Sugiura. 2000a. Evaluation of RFLP analysis for discriminating PCNA genotype in some persimmon cultivars. J. Japan. Soc. Hort. Sci. 69: 702-704.

Kanzaki, S., K. Yonemori, A. Sato, M. Yamada and A. Sugiura. 2000b. Analysis of the genetic relationships among pollination-constant and non-astringent (PCNA) cultivars of persimmon (Diospyros kaki Thunb.) from Japan and China using amplified fragment length polymorphism (AFLP) J. Japan. Soc. Hort. Sci. 69: 665-670.

Kanzaki, S., K. Yonemori, A. Sugiura, A. Sato and M. Yamada. 2001. Identification of molecular markers linked to the natural astringency-loss of Japanese persimmon (Diospyros kaki Thunb.) fruit. J. Amer. Soc. Hort. Sci. 126: 51-55.

Kriegner, A., J. C. Cervantes, K. Burg, R. O. M. Mwanga and D. P. Zhang. 2003. A genetic linkage map of sweetpotato [Ipomoea batatas (L.) Lam.] based on AFLP markers. Mol. Breeding 11: 169-185.

Michelmore, R. W., I. Paran and R. V. Kesseli. 1991. Identification of markers linked to disease-resistance genes by bulked segregant analysis: A rapid method to detect markers in specific genome regions by using segregation population. Proc. Natl. Acad. Sci. USA 88: 9828-9832.

Sugiura, A. and T. Tomana. 1983. Relationships of ethanol production by seeds of different types of Japanese persimmons and their tannin content. HortScience 18: 319321.

Tanaka, T., R. Takahashi, I. Kouno and G. Nonaka. 1994. Chemical evidence for the de-astringency (insolubilization of tannins) of persimmon fruit. J. Chem. Sco. Perkin Trans. 1:3013-3022.

Vos, P., R. Hogers, M. Bleeker, M. Reijans, T. Lee, M. Hornes, A. Frijters, J. Pot, J. Peleman, M. Kuiper and M. Zabeau. 1995. AFLP: A new technique for DNA fingerprinting. Nucl. Acid Res. 23: 4407-4414.

Wang, R. 1982. The origin of 'Luo Tian Tian Shi'. Chinese Fruit Tree 2: 16-19 (In Chinese).

Yamada., M. and A. Sato. 2002. Segregation for fruit astringency type in progenies derived from crosses of 'Nishimurawase' $\times$ pollination constant nonastringent genotypes in Oriental persimmon (Diospyros kaki Thunb.). Sci. Hortic. 92: 107111.

Yonemori, K. and J. Matsushima. 1985. Property of development of the tannin cells from nonastringnet and astringent type fruits of Japanese persimmon (Diospyros kaki) and its relationship to natural astringency. J. Japan. Soc. Hort. Sci. 54: 201-208 (In Japanese with English abstract).

Yonemori, K. and J. Matsushima. 1987. Changes in tannin cell morphology with growth and development of Japanese persimmon fruit. J. Amer. Soc. Hort. Sci. 112: 818-821.

Yonemori, K., A. Ikegami, A. Kitajima, Z. Luo, S. Kanzaki, A. Sato, M. Yamada and Y. Wang. 2005. Existence of several pollination constant non-astringent type persimmons in China. Acta Hort. 685: 77-84.

Yonemori, K., A. Sugiura and M. Yamada. 2000. Persimmon genetics and breeding. Plant Breed. Rev. 19: 191-225. 